\title{
A NONEXISTENCE RESULT FOR MOORE $G$-SPECTRA
}

\author{
S. R. COSTENOBLE AND S. WANER
}

(Communicated by Frederick R. Cohen)

\begin{abstract}
In this paper we show that certain equivariant Moore spectra do not exist. Specifically, we give an example of a Bredon coefficient system for which there is no corresponding equivariant Moore CW-spectrum that is bounded below. This nonexistence result is stronger than nonexistence results shown previously; nonexistence of an equivariant Moore spectrum of type $T$ implies, in particular, that there are no equivariant Moore spaces of type $(T, n)$ for any $n$. As a key step, we show that there is no strictly commutative Hopf space structure on the loop space $Q S^{0}$ agreeing up to infinite loop homotopy with the usual addition.
\end{abstract}

\section{INTRODUCTION}

We wish to show that certain equivariant Moore spectra do not exist. First we must explain what we mean by a Moore spectrum. Let $G$ be a compact Lie group. A $G$-spectrum for us will be a $G$-spectrum indexed on a universe with $G$-trivial action, in the sense of [LMS]. These are the spectra that represent integer graded Bredon equivariant homology and cohomology theories [B]. A fundamental example is $H \mathbb{Z}$, the nonequivariant Eilenberg-MacLane spectrum given trivial $G$-action. This represents ordinary equivariant cohomology with constant $\mathbb{Z}$ coefficients, in the sense of Bredon. Moore spectra can be used to introduce other coefficient systems into ordinary equivariant cohomology, as follows. Recall from [B] that a coefficient system is a contravariant functor from $h \mathscr{G}$, the homotopy orbit category of $G$, to the category of abelian groups. If $T$ is a coefficient system, we seek to find a spectrum $M$ such that $M \wedge H \mathbb{Z}$ represents ordinary $G$-cohomology with $T$ coefficients. This means that we should have, for each subgroup $K$ of $G$,

$$
(M \wedge H \mathbb{Z})^{*}(G / K) \cong T(G / K)
$$

concentrated in degree 0 , and this should be an isomorphism of coefficient systems as $K$ varies. Such a spectrum $M$ will be called a Moore spectrum of

Received by the editors December 7, 1989 and, in revised form, March 22, 1990.

1980 Mathematics Subject Classification (1985 Revision). Primary 55P99, 57S17, 55P62, 55Q05.

Key words and phrases. Infinite loop maps, equivariant, Moore spectra, Moore spaces, coefficient system. 
type $T$. Notice also that

$$
(M \wedge H \mathbb{Z})^{-n}(G / K)=h G \mathscr{S}\left(\Sigma^{n} G / K^{+} \wedge S, M \wedge H \mathbb{Z}\right) \cong H_{n}\left(M^{K} ; \mathbb{Z}\right),
$$

where $S$ is the sphere spectrum and $h G \mathscr{S}(-,-)$ denotes stable $G$-maps between $G$-spectra indexed on a $G$-trivial universe. Therefore, $M$ is a Moore spectrum of type $T$ if and only if $M^{K}$ is a nonequivariant Moore spectrum of type $T(G / K)$ for each $K$, and if the map $M^{J} \rightarrow M^{K}$ induced by a map $G / K \rightarrow G / J$ induces the homomorphism $T(G / J) \rightarrow T(G / K)$ in homology.

Moore spectra are used more generally to endow any generalized homology or cohomology theory with coefficients, and for this to be useful, one must have an associated universal coefficients spectral sequence. In this regard, even nonequivariantly, Moore spectra which fail to be bounded below are inappropriate, as the expected universal coefficients spectral sequence result (short exact sequence in the nonequivariant case) fails in general. We may illustrate this by means of a simple example: Take $B U \mathbb{Z}_{2}$ to be the spectrum underlying periodic $K$-theory with $\mathbb{Z}_{2}$ coefficients. Then it is well-known that $H_{*}\left(B U \mathbb{Z}_{2}\right)=0$, so that $M=B U \mathbb{Z}_{2} \vee M \mathbb{Z}_{3}$ is a Moore CW-spectrum of type $\mathbb{Z}_{3}$, where we take $M \pi$ to be any connective Moore spectrum of type $\pi$. For the universal coefficients result to apply to the cohomology theory $(M \wedge E)^{*}(-)$, where $E$ is any spectrum, one must have a short exact sequence

$$
0 \rightarrow E^{n}(X) \otimes \mathbb{Z}_{3} \rightarrow(M \wedge E)^{n}(X) \rightarrow \operatorname{Tor}_{1}\left(E^{n+1}(X), \mathbb{Z}_{3}\right) \rightarrow 0
$$

for finite CW-complexes $X$. Taking $X=S^{0}$, and $E=M \mathbb{Z}_{2}$, one obtains $E^{*}(X) \otimes \mathbb{Z}_{3}=0$ and $\operatorname{Tor}_{1}\left(E^{*}(X), \mathbb{Z}_{3}\right)=0$, whereas $(M \wedge E)^{*}(X) \cong\left(B U \mathbb{Z}_{2}\right)^{*}$ $\left(S^{0}\right) \neq 0$.

We therefore take the view that the useful Moore CW-spectra are those that are bounded below, and we shall give an example, with $G$ the dihedral group of order 8 , of a coefficient system $T$ for which there exists no bounded-below Moore CW-spectrum of type $T$. We do not know whether there exists a Moore spectrum of type $T$ if we drop the boundedness requirement.

Similar questions have been considered by various people. Steenrod's Problem asks for a $G$-space $X$ such that $H_{*}(X)$ is a given $G$-module concentrated in degree $n$. There are a number of existence and nonexistence results $($ see $[\mathrm{K}]$ for an existence result, and further references). As far as equivariant homotopy theory is concerned, it is more useful to take into account the behavior of all of the fixed-sets. Again, there have been various results about the existence or uniqueness of equivariant Moore spaces in this sense $([\mathrm{K}, \mathrm{T}])$, particularly when working rationally. The nonexistence result we prove here is stronger than the nonexistence results shown previously; showing that there is no equivariant bounded-below Moore spectrum of type $T$ implies, in particular, that there are no equivariant Moore spaces of type $(T, n)$ for any $n$.

In a future paper we hope to approach the problem more generally, showing that equivariant connective Moore spectra always exist for rational coeffi- 
cient systems, and developing a general obstruction theory for the existence of bounded-below Moore spectra.

This paper is organized as follows: In $\S \S 2$ and 3 we do some homotopy theory and some geometry to show that the usual addition on $Q S^{0}$ cannot be made strictly commutative. (It has been previously shown [M1, 3.6, M3] that it cannot be made strictly unital, commutative, and associative simultaneously.) Section 4 then gives an example of a coefficient system which, if realized by a boundedbelow Moore spectrum, would produce just such a commutative addition.

The authors are grateful to the reviewer of an earlier draft of this paper for useful criticism, and in particular for pointing out that our methods apply only to spectra that are bounded below.

\section{2. НомОтоРY}

Here we derive a few elementary results on the homotopy theory of nonequivariant infinite loop maps. We refer the reader to [M2] for a treatment of the theory of nonequivariant spectra.

By way of notation, $Q S^{0}$ will denote the infinite loop space $\operatorname{colim}_{n} \Omega^{n} S^{n}$, and the sphere spectrum (whose 0th space is $Q S^{0}$ ) will be denoted by $S$. The homotopy category of spectra and maps between spectra is denoted by $h \mathscr{S}$.

Lemma 2.1. Let $f: Q S^{0} \rightarrow Q S^{0}$ be an infinite loop map such that $f$ maps the degree 1 component to itself. Then $f$ is homotopic to the identity as an infinite loop map.

Proof. Since homotopy classes of infinite loop maps $Q S^{0} \rightarrow Q S^{0}$ are in 1-1 correspondence with homotopy classes of maps of spectra $S \rightarrow S$, the result follows from the isomorphism $h \mathscr{S}(S, S) \cong \mathbb{Z}$.

As a consequence of this, we obtain

Lemma 2.2. Let $f$ and $g$ be maps $S \times S \rightarrow S$ whose restrictions to zeroth spaces agree on components. Then $f$ and $g$ are homotopic.

Proof. One has

$$
h \mathscr{S}(S \times S, S) \cong h \mathscr{S}(S \vee S, S) \cong h \mathscr{S}(S, S) \oplus h \mathscr{S}(S, S) \cong \mathbb{Z} \oplus \mathbb{Z},
$$

and the result follows from Lemma 2.1 .

Lemma 2.3. If $f$ and $g$ are any two homotopic maps $S \times S \rightarrow S$, then there are four homotopy classes of homotopies from $f$ to $g$.

Proof. Without loss of generality, we can assume $f=g$. Further, by translation, we can assume that $f$ is trivial, since homotopy classes of maps between spectra form a group. Thus we consider

$$
\begin{aligned}
h \mathscr{S}(\Sigma(S \times S), S) & \cong h \mathscr{S}(\Sigma(S \vee S), S) \cong h \mathscr{S}(\Sigma S \vee \Sigma S, S) \\
& \cong h \mathscr{S}(\Sigma S, S) \oplus h \mathscr{S}(\Sigma S, S) \cong \mathbb{Z}_{2} \oplus \mathbb{Z}_{2},
\end{aligned}
$$

showing the assertion. 
Corollary 2.4. If $f$ and $g$ are any two homotopic infinite loop maps $Q S^{0} \times$ $Q S^{0} \rightarrow Q S^{0}$, then there are four homotopy classes of paths from $f$ to $g$ through infinite loop maps.

From the proof of Lemma 2.3 we can see that, when $f=g=*$, these four classes are given by the trivial homotopy $D_{1}$ and the composites

$$
T_{i}: \Sigma\left(Q S^{0} \times Q S^{0}\right) \stackrel{\Sigma p_{i}}{\longrightarrow} \Sigma Q S^{0} \stackrel{H}{\longrightarrow} Q S^{0}
$$

and

$$
D_{2}: \Sigma\left(Q S^{0} \times Q S^{0}\right) \stackrel{\Sigma \mu}{\longrightarrow} \Sigma Q S^{0} \stackrel{H}{\longrightarrow} Q S^{0}
$$

where $p_{i}$ are the projections, $\mu$ is the usual addition, and $H$ is the Hopf map. It then follows that, if $f$ and $g$ are any two homotopic infinite loop maps $Q S^{0} \times Q S^{0} \rightarrow Q S^{0}$ with $K$ an infinite loop homotopy between them, then one can obtain the homotopy classes of all infinite loop homotopies as follows: First we define representatives of the four classes of homotopies of $f$ with itself as the composites

$$
Q S^{0} \times Q S^{0} \times I \stackrel{\Delta \times 1}{\longrightarrow} Q S^{0} \times Q S^{0} \times Q S^{0} \times Q S^{0} \times I \stackrel{f \times J}{\longrightarrow} Q S^{0} \times Q S^{0} \stackrel{\mu}{\longrightarrow} Q S^{0} .
$$

Here, $\Delta$ is the diagonal on $Q S^{0} \times Q S^{0}$ and $J$ is a representative of one of the four homotopy classes described above. We can now follow these homotopies with the homotopy $K$ to obtain the four classes of homotopies from $f$ to $g$. We shall refer to these four classes as $D_{i}(K)$ and $T_{i}(K)$ for $i=1,2$, according as $J=D_{i}$ or $T_{i}$.

\section{GeOMETRY}

Here we use geometric arguments to prove the following theorem.

Theorem 3.1. If $\nu$ is an infinite loop map $Q S^{0} \times Q S^{0} \rightarrow Q S^{0}$ which agrees up to infinite loop homotopy with the usual addition, then $\nu$ cannot be strictly commutative.

In [M1], May describes a map from the space of configurations of little cubes in the $n$-cube $I^{n}$ to $\Omega^{n} S^{n}$. Caruso and Waner [CW] extend this to the space of configurations of signed cubes in $I^{n}$, where a positive and a negative cube of the same size are allowed to merge and cancel only along the direction of $I \times\{0\}^{n-1} \subset I^{n}$. (See Figure 1, which shows two cubes in $I^{3}$ in the process of merging and cancelling in the vertical direction.) The map into $\Omega^{n} S^{n}$ is given by the usual Pontryagin-Thom collapse map, so that the merging of positive and negative little cubes corresponds to the usual cancelling of a degree 1 and a degree -1 map $S^{n} \rightarrow S^{n}$. This easily extends to the stable case by taking colimits over $n$. Although it is shown in [CW] that this gives a homotopy equivalence, we shall merely use it as a device to describe specific paths in $Q S^{0}$.

We define paths in $Q S^{0}$ using braids of positive and a negative strands in $I^{n} \times I$, whose intersection with each slice $I^{n} \times\{t\}$ is a configuration of positive 

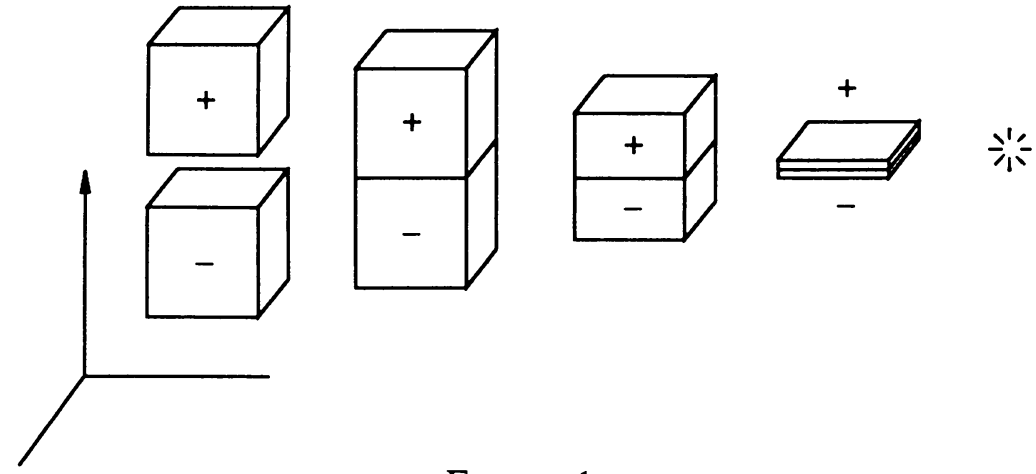

FIGURE 1

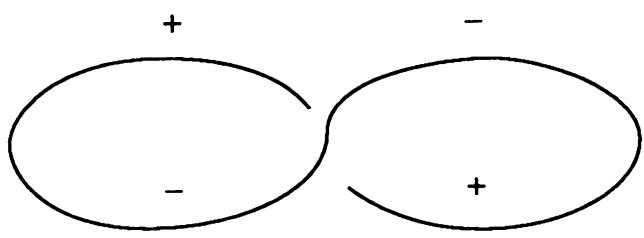

FIGURE 2.0

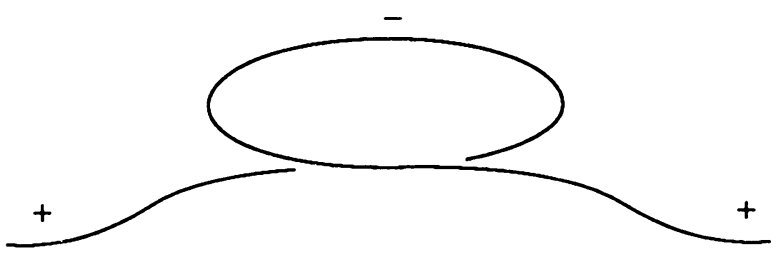

FIGURE 2.1

and negative cubes. A positive and negative strand are allowed to cancel in the direction $D$ of $I \times\{0\}^{n-1} \times\{0\} \subset I^{n} \times I$; using the Pontryagin-Thom collapse map on each slice $I^{n} \times\{t\}$ gives a map from $I$ into $Q S^{0}$. As an example, consider the diagrams of Figure 2, representing positive and negative strands in $I^{2} \times I$. Here, the direction $D$ is vertical while the last variable $t$ runs from left to right. The lines drawn should be thought of as "fattened up," so that a slice perpendicular to the $t$ direction produces a configuration of little cubes (squares in these pictures). Figure 3 (p. 270) shows six of these slices through Figure 2.0. Using physics terminology, Figure 2.0 represents the creation and annihilation of a "virtual pair" of cubes; it represents a loop in the degree 0 component of $Q S^{0}$, starting and ending at the trivial map $S^{n} \rightarrow S^{n}$. However, as is about to be shown, the twisting in the middle makes this loop essential.

Lemma 3.2. The diagram in Figure 2.2 represents an essential loop in the degree 2 component of $Q S^{0}$. 


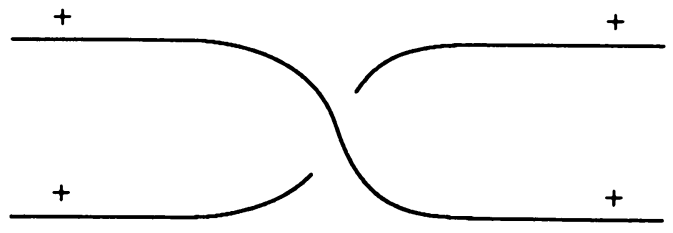

FIGURE 2.2
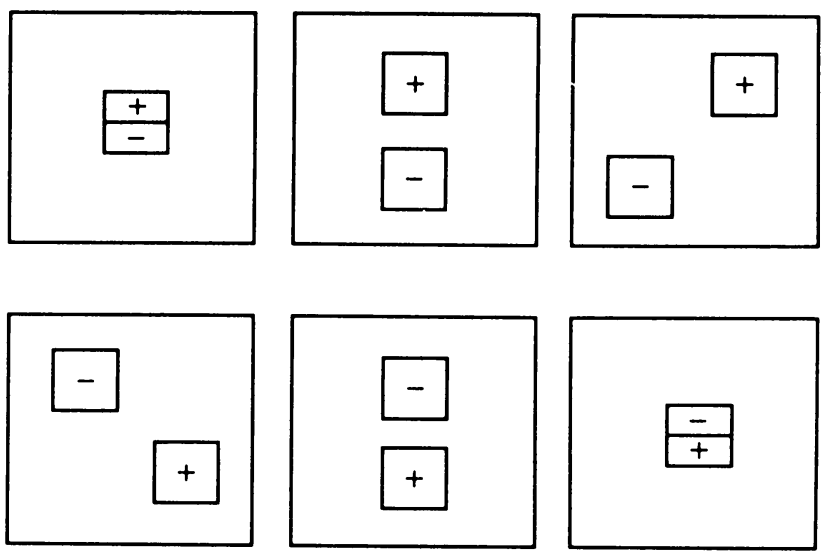

FIGURE 3

Proof. First note that the diagram describes a loop in the space $C_{2}$ of unordered pairs of little cubes. Since $C_{2}$ is the orbit space of a free $\mathbb{Z}_{2}$-action on a contractible space (the space of ordered pairs of little cubes), $C_{2}$ may be identified with $B \Sigma_{2} \cong \mathbb{R} P^{\infty}$; this map may be realized explicitly by taking a pair of little cubes to the unique line through the origin parallel to the line through their centers. The given loop is then clearly carried to the nontrivial element $h$ of $\pi_{1}\left(\mathbb{R} P^{\infty}\right)$. Further, the Pontryagin-Thom map $\gamma: C_{2} \rightarrow Q S^{0}$ extends to a group completion $\amalg_{n \geq 0} C_{n} \rightarrow Q S^{0}$ [M1, 8.11 and 8.14], so that the element $h$ is taken to the nonzero element in the fundamental group of the 2 component of $Q S^{0}$.

Corollary 3.3. The diagrams in Figures 2.0, 2.1, and 2.2 represent essential loops in the degree 0,1 , and 2 components of $Q S^{0}$ respectively; the first of these represents the Hopf map and the others its translates.

Proof. We refer to the sequence of diagrams in Figure 4. The first diagram represents the translate of the diagram in Figure 2.0 to the degree 2 component of $Q S^{0}$, and the subsequent diagrams represent successive stages in a homotopy with the loop of Figure 2.2. Notice the appearance at the fourth stage of this homotopy of a translate of the diagram in Figure 2.1. Since the Hopf map is the only essential loop in the degree 0 component of $Q S^{0}$, this is what Figure 2.0 must be. 

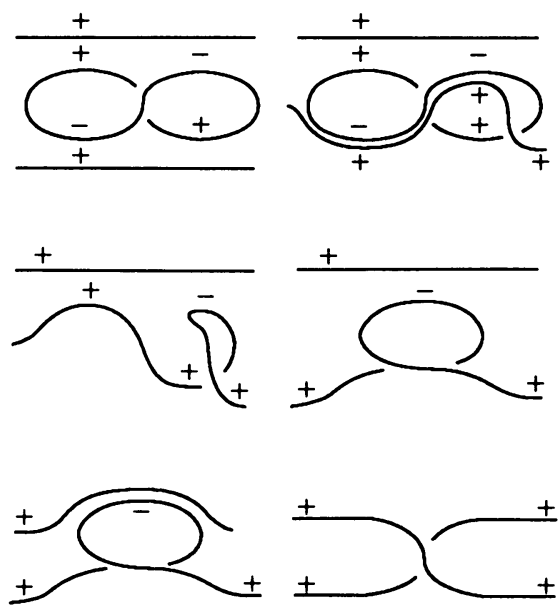

FIGURE 4

Before proving Theorem 3.1, we should comment on the map $H: \Sigma Q S^{0} \rightarrow$ $Q S^{0}$ we used in $\S 2$. If we take a configuration of little cubes, giving a point $c \in Q S^{0}$, we can form the composite $I \rightarrow Q S^{0} \times I \rightarrow Q S^{0}$, where the first map is $t \mapsto(c, t)$ and the second is $H$. This composite will be a loop in the degree 0 component of $Q S^{0}$ starting and ending at the trivial map, and will be represented by the braid having parallel copies of the diagram of Figure 2.0, one for each cube in the original configuration. This follows by first looking at the special case where $c$ is the constant map in the degree 1 component; then the composite loop is exactly the Hopf loop $\Sigma S^{n} \rightarrow S^{n}$, which we have just shown is represented by Figure 2.0. In general, write any configuration as a sum of copies of the identity map or its inverse, using the fact that addition in the little cubes model is just juxtaposition.

Proof of Theorem 3.1. Assume given a strictly commutative infinite loop map $\nu: Q S^{0} \times Q S^{0} \rightarrow Q S^{0}$ as in the hypothesis. Let $\mu$ denote the usual addition on $Q S^{0}$, and let $\tau$ denote the twist map on $Q S^{0} \times Q S^{0}$. Define a homotopy $\Psi: \mu \simeq \mu \circ \tau$ in two stages; for the first stage, use a given homotopy $\Phi: \mu \simeq \nu$, and for the second stage, use $\Phi^{\prime} \circ \tau$, where $\Phi^{\prime}$ is $\Phi$ reversed. By $\S 2, \Psi$ must be homotopic with one of four possible homotopies $Q S^{0} \times Q S^{0} \times I \rightarrow Q S^{0}$. Let $\Gamma$ denote the usual homotopy $\mu \simeq \mu \circ \tau$; applied to the sum of two little cubes it gives the picture of Figure 2.2.

Case 1. $\Psi \simeq D_{1}(\Gamma)$ or $D_{2}(\Gamma)$. Consider the composite

$$
\lambda: I \stackrel{\omega}{\longrightarrow} Q S^{0} \times Q S^{0} \times I \stackrel{\Psi}{\longrightarrow} Q S^{0},
$$

where $\omega(t)=(l, l, t)$ with $l$ the identity element of $Q S^{0} . \lambda$ describes a loop in the degree 2 component of $Q S^{0}$ which, by construction of $\Psi$ and the fact that $(\tau \times 1) \circ \omega=\omega$, is necessarily null-homotopic. On the other hand, using 


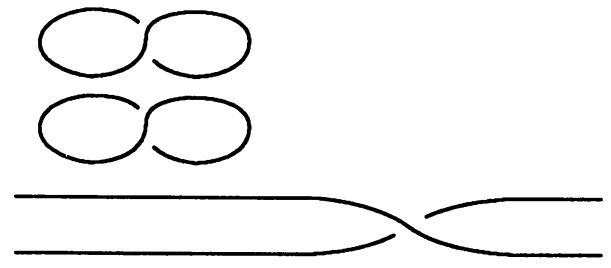

FIGURE 5

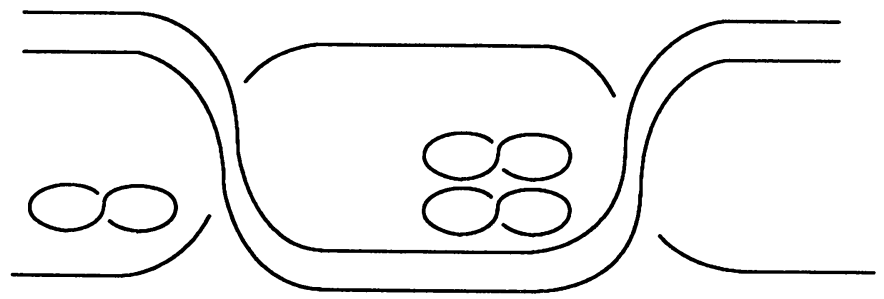

FIGURE 6

$D_{1}(\Gamma)=\Gamma$ in place of $\Psi$ produces the loop of Figure 2.2, which is essential by Proposition 3.2. Using $D_{2}(\Gamma)$ in place of $\Psi$ would produce the loop of Figure 5 , which is again a translate of the Hopf map. (Notice that the "figure eights," being copies of the Hopf map, cancel each other.)

Case 2. $\Psi \simeq T_{1}(\Gamma)$ or $T_{2}(\Gamma)$. Define two paths $\lambda_{1}$ and $\lambda_{2}$ as the composites

$$
\lambda_{i}: I \stackrel{\omega_{i}}{\longrightarrow} Q S^{0} \times Q S^{0} \times I \stackrel{\Psi}{\longrightarrow} Q S^{0},
$$

where $\omega_{1}(t)=(l, \theta, t)$ with $l$ as above and $\theta$ the degree 2 map represented by two little cubes. $\omega_{2}$ is similarly defined by $\omega_{2}(t)=(\theta, l, t)$. The path sum $\phi=\lambda_{1} \# \lambda_{2}$ is a null-homotopic loop by the construction of $\Psi$ and the fact that $(\tau \times 1) \circ \omega_{1}=\omega_{2}$. On the other hand, using $T_{1}(\Gamma)$ or $T_{2}(\Gamma)$ in place of $\Psi$ would produce an essential loop. We refer the reader to Figure 6 for a description of $\phi$ in the case $\Psi=T_{1}(\Gamma)$, the figure for $T_{2}(\Gamma)$ being similar.

\section{Nonexistence of general Moore $G$-SPeCtra}

Referring to the Introduction for the relevant definitions, we now produce the promised example. Let $G$ be the dihedral group of order 8: $G=\langle a, b| a^{4}=$ $\left.1=b^{2}, b a=a^{3} b\right\rangle$. Its orbit lattice $\mathscr{G}$ is shown in Figure 7 , with some multiple arrows suppressed.

Notice that $G /\left\langle a^{2}, b\right\rangle$ has $\mathbb{Z}_{2}$ as its group of self-maps, as does $G /\langle b\rangle$; there are two maps from $G /\langle b\rangle$ to $G /\left\langle a^{2}, b\right\rangle$, with the group of self-maps of $G /\left\langle a^{2}, b\right\rangle$ acting nontrivially, and the group of self-maps of $G /\langle b\rangle$ acting trivially.

Define a coefficient system $T$ on $\mathscr{G}$ by the lattice of coefficient groups shown in Figure 8. Here, the map labeled 2 is multiplication by $2, \tau$ is the twist map, $\Delta$ the diagonal, $s$ the sum map and $p_{i}$ the two projections (i.e., of the two maps $T\left(G /\left\langle a^{2}, b\right\rangle\right) \rightarrow T(G /\langle b\rangle)$, one is $p_{1}$, projection to the first summand, 


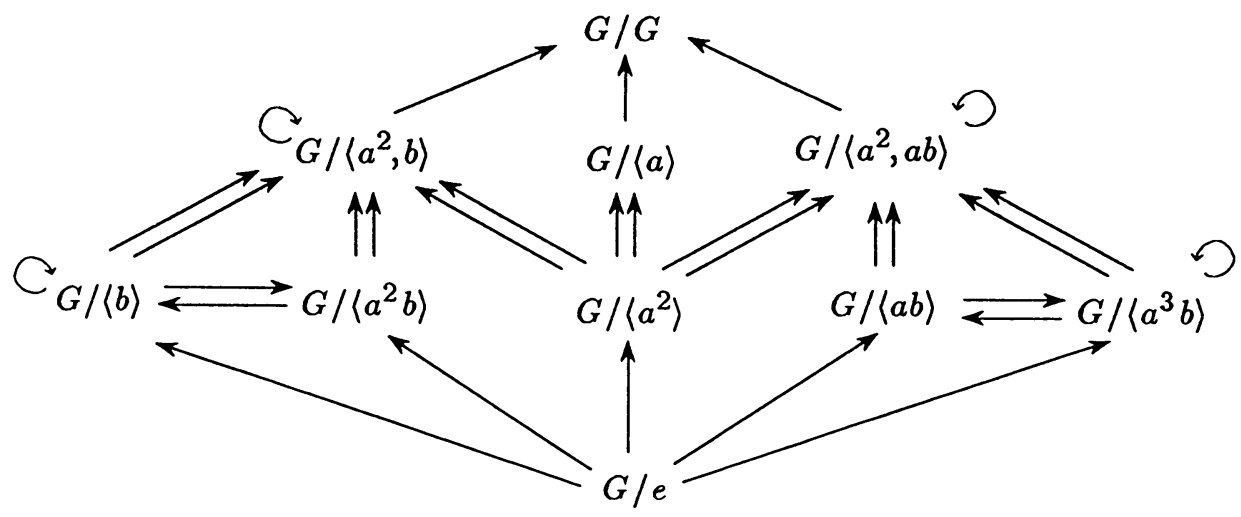

FIGURE 7

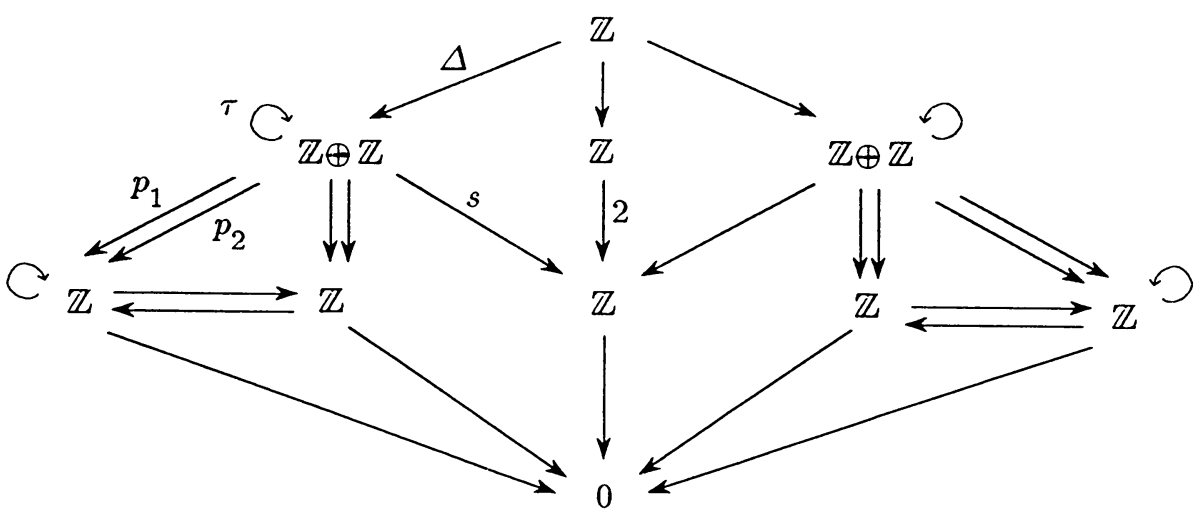

Figure 8

and the other is $p_{2}$, projection to the second summand). All unlabeled maps from $\mathbb{Z}$ to $\mathbb{Z}$ are identity maps, and the diagram is symmetric.

Theorem 4.1. There is no equivariant Moore spectrum of type $T$ that is bounded below.

Proof. Assume there is such a $G$-spectrum $M$. Since $M$ is bounded below, so are all of its fixed set spectra. This allows us to say that a homology equivalence is a homotopy equivalence. Let $H=\left\langle a^{2}, b\right\rangle, K=\langle b\rangle$, and $L=\left\langle a^{2}\right\rangle$. We then have the following homotopy equivalences of spectra:

$$
\begin{aligned}
& M^{G} \simeq S ; \\
& M^{H} \simeq S \times S ; \\
& M^{K} \simeq M^{L} \simeq S .
\end{aligned}
$$

Notice that the action of $\tau$ defines a $\mathbb{Z}_{2}$-action on $M^{H}$. We claim that the 
second equivalence above may be arranged to be an equivariant homotopy equivalence, where $\mathbb{Z}_{2}$ acts on $S \times S$ by permuting the factors. Define a map $f: M^{H} \rightarrow M^{K} \times M^{K}$ as the pair $\left(l_{1}, l_{2}\right)$, where $l_{1}$ and $l_{2}$ are the maps induced by the two $G$-maps from $G / K$ to $G / H$. That this is an equivariant map follows from the functoriality of the fixed-set construction. Further, $f$ is a nonequivariant equivalence since it is required to induce an equivalence in homology. The $\mathbb{Z}_{2}$-fixed set of $M^{H}$ is $M^{G}$, and the restriction of $f$ to the fixed set is $f^{G}: M^{G} \rightarrow \Delta\left(M^{K} \times M^{K}\right) \simeq M^{K}$ where $\Delta$ is the diagonal, and where we may take the isomorphism to be one of the projections. Since this composite coincides with inclusion of $M^{G}$ in $M^{K}$, it is an equivalence in homology, and hence a homotopy equivalence. That $f$ is an equivariant equivalence now follows from the equivariant Whitehead theorem applied to $G$-spectra [LMS]. We conclude that $M^{H} \simeq S \times S$ by the $\mathbb{Z}_{2}$-equivariant equivalence $M^{K} \times M^{K} \simeq S \times S$.

Now define a $\mathbb{Z}_{2}$-equivariant map $\nu: S \times S \rightarrow S$, where the action on $S$ is the trivial one, as the composite

$$
S \times S \simeq M^{H} \stackrel{\sigma}{\longrightarrow} M^{L} \simeq S,
$$

where $\sigma$ is induced by the projection $G / L \rightarrow G / H$. In homology, the map $\nu$ coincides with the usual addition $H_{*}(S \times S) \cong H_{*}(S \vee S) \rightarrow H_{*}(S)$; by Lemma 2.2 it is homotopic with the usual addition $\mu$. Restricting to zeroth spaces now gives a strictly commutative map $\nu: Q S^{0} \times Q S^{0} \rightarrow Q S^{0}$ which is homotopic as an infinite loop map with the usual addition, contradicting Theorem 3.1.

\section{REFERENCES}

[B] G. E. Bredon, Equivariant cohomology theories, Lecture Notes in Math., vol. 34, SpringerVerlag, Berlin and New York, 1967.

[CW] J. Caruso and S. Waner, An approximation to $\Omega^{n} \Sigma^{n} X$, Trans. Amer. Math. Soc. 265 (1981), 147-162.

[K] P. J. Kahn, Rational Moore G-spaces, Trans. Amer. Math. Soc. 298 (1986), 245-271.

[LMS] G. Lewis Jr., J. P. May and M. Steinberger, Equivariant stable homotopy theory, Lecture Notes in Math., vol. 1213, Springer-Verlag, Berlin and New York, 1986.

[M1] J. P. May, Geometry of iterated loop spaces, Lecture Notes in Math., vol. 271, SpringerVerlag, Berlin and New York, 1972.

[M2] _,$E^{\infty}$ ring spaces and $E^{\infty}$ ring spectra and their applications, Lecture Notes in Math., vol. 577, Springer-Verlag, Berlin and New York, 1977.

[M3] J. Moore, Symposium internacional de topologia algebraica, Univ. Nac. Auton. Mexico (1958), 232-247.

[T] G. Triantafillou, G-spaces with prescribed equivariant cohomology, Univ. of Minnesota, preprint. 